\title{
Distribution of First Passage Times for Lumped States in Markov Chains
}

\author{
Murat Gül ${ }^{1}$ and Salih Çelebioğlu ${ }^{2}$ \\ 1. Department of Statistic, Giresun University, Güre, Giresun, Turkey. \\ 2. Department of Statistic, Gazi University, Teknikokullar, Ankara, Turkey.
}

Received: April 02, 2014 / Accepted: April 29, 2014 / Published: August 25, 2015.

\begin{abstract}
First passage time in Markov chains is defined as the first time that a chain passes a specified state or lumped states. This state or lumped states may indicate first passage time of an interesting, rare and amazing event. In this study, obtaining distribution of the first passage time relating to lumped states which are constructed by gathering the states through lumping method for a irreducible Markov chain whose state space is finite was deliberated. Thanks to lumping method the chain's Markov property has been preserved. Another benefit of lumping method in the way of practice is reduction of the state space thanks to gathering states together. As the obtained first passage distributions are continuous, it may be used in many fields such as reliability and risk analysis
\end{abstract}

Key words: Markov chain, distribution of first passage time, lumped states.

\section{Introduction}

The first-passage concept used in many areas from controlled reactions in physical and chemical processes to chromatographic, stochastic processes play an important role. Therefore, you need to know the first passage characteristics to understand the movements of real systems. Once this connection is established, it is quite simple to obtain the dynamical properties of the system in terms of well-known first-passage. The problems associated with these systems can be found in [1-5].

The first passage time variable can have different meanings in different areas. For classical gambler's ruin type models this variable can represent the duration of the game, for physics this variable is the time period for a particle in a system reaching some state, for reliability engineering studies the expectation of this variable corresponds to the mean time for the first failure and its survivor function is regarded as the reliability of a system under study.

Corresponding author: Murat Gül, Department of Statistic, Giresun University, Turkey. E-mail: murat.gul@giresun.edu.tr.
Passage times have been investigated since early days of probability theory and its applications. The best known example is the first passage/entrance time to a set, which embraces waiting times, absorption problems, extinction phonomena, busy periods and other applications.

Although passage times are in fact examples of stopping times, they enjoy important position in theoretical and practical applications. Therefore, it is possible to encounter the first passage time applications in many areas of science and engineering.

Kammer [6] used the first passage time process for the credit risks of firms and proposed to define the threshold level as a function of the firm's liabilities.

$\mathrm{Li}$ and Shaked [7] cited a stochastic process as the damage level of a certain device at $t$ time and defined the first passage time as a time of exceeding the failure rate of a device subjected to shocks and wear .They studied the features of first passage times which had increasing failure rate and increasing failure rate average.

Mandel [8] analyzed the phases of sclerosis by using properties of Markov chain and by using 
transition matrix, he estimated the mean first passage time to various phases of the disease for he treated and untreated. Thus, opportunity of describing and predicting the course of the disease came up.

On the other hand, the lumping of Markov processes is one such very useful technique. When the number of states increases, Markov problems grows rapidly. So large scale Markov models are intractably hard to solve. Under certain conditions the state space of a discrete parameter finite Markov chain may be partitioned into subsets, each of which may be treated as a single state of a smaller chain that retains the Markov property.

Lumped states which are constructed by gathering the states through lumping method for a irreducible Markov provide a macroscopic view of original states

The stochastic processes literature is abuntant with several papers, and a book by Kemeny and Snell, which exploit lumpability of the discrete-time Markov processes or chain.

Ridder [9], for simulate a rare event problem, the correspondence between the cross-entropy method and the zero-variance approximation showed. In a rare event problem, large state space has been partitioned into three sets: "good states", "failed or bad states", "internal states" when he is constructing Markov model. Ridder [9] interested in first passage time probability that Markov chain will pass the failure set before the good set when the chain starts in internal state

Tian and Kannan [10] extend this notion of lumpability to continuous time finite state space Markov chains and characterize lumpability in terms of the infinitesimal generator. Under suitable conditions they show to recover some of the basic quantities such as mean first return time, mean absorbing time of the original Markov process from the jump chain of the lumped Markov process.

In this study, higher the number of state is in a Markov chain with finite state space, the more difficult a transaction is, and hence it is an important problem that should be worked to define the way how to approach through a continuous distribution for the discrete first passage time distribution based on the lumping method that allows reducing the number of state, and facilitates calculation in the modelling.

This study emphasizes the solution of this problem. As the obtained first passage distributions are continuous, it may be used in many fields such as reliability and risk analysis.

\section{Methods}

In this section, we will summarize the methodology used for calculating first passage times for Markov chain.

\subsection{Markov Chain}

In this process, the outcome of a given experiment can affect the outcome of the next experiment. In other words, Markov chains are the stochastic processes whose futures are conditionally independent of their pasts provided that their present values are known.

Let $\left\{X_{n}: n=0,1,2, \ldots\right\}$ be a stochastic process that has a finite or countable infinite state space $S$. When $X_{n}=i$ we say that 'the process is in state $i$ at time $n$. The probability that the process is in state $j$ in the next time provided that its present state is $i$, is denoted by $P_{i j}$.

For the Markov chains, the transition probabilities are arranged in a matrix form and the resulting matrix is called the transition matrix of the chain. The elements of a transition matrix hold the following conditions:

a) for any two states $\mathrm{i}, \mathrm{j} \in \mathrm{S}, P_{i j} \geq 0$; and

b) for all $i \in S, \quad \sum_{j} P_{i j}=1$.

It is often desirable to also make probability statements about the number of transitions made by the process in going from state $i$ to state $\mathrm{j}$ for the first time. This length of time is called the first passage time in going from state $i$ to state $j$. 
To illustrate these definitions, reconsider the inventory example where $X_{t}$ is the number of cameras on hand at the end of week $t$, where we start with $X_{0}$. Suppose that it turns out that

$$
X_{0}=3, X_{1}=2, X_{2}=1, X_{3}=0, X_{4}=3, X_{5}=1
$$

In this case, the first passage time in going from state 3 to state 1 is 2 weeks, the first passage time in going from state 3 to state 0 is 3 weeks, and the recurrence time for state 3 is 4 weeks [11].

In general, the first passage times are random variables. The probability distributions associated with them depend upon the transition probabilities of the process. In particular, let $f_{i j}^{(n)}$ denote the probability that the first passage time from state $i$ to $j$ is equal to $n$. For $n>1$, this first passage time is $n$ if the first transition is from state $i$ to some state $k$ $(k \neq j)$ and then the first passage time from state $k$ to state $j$ is $n-1$. Therefore, these probabilities satisfy the following recursive relationships [12] .

$$
\begin{aligned}
f_{i j}^{(1)} & =p_{i j}^{(1)}=p_{i j}, \\
f_{i j}^{(2)} & =\sum_{k \neq j} p_{i k} f_{k j}^{(1)}, \\
f_{i j}^{(n)} & =\sum_{k \neq j} p_{i k} f_{k j}^{(n-1)}
\end{aligned}
$$

In analyzing and using Markov chain, first passage times are fundamental to understanding the long-run behavior of a Markov chain [13]. Among the Markov chain characteristics, the first passage times play an important role. For any two states, the first passage time probability in $n$ steps is defined as follows and this probability is related to the ever reaching probability.

Definition 2.1.1: For any two states $i$ and $j$, the first passage time probability from $i$ to $j$ in $n$ steps,

$f_{i j}^{(n)}$ is defined as

$$
f_{i j}^{(n)}=\left\{\begin{array}{l}
p_{i j} ; n=1 \\
\sum_{k \neq j} p_{i k} f_{k j}^{(n-1)} ; n=2,3, \ldots
\end{array}\right.
$$

\subsection{Lumpability Theory}

Obtained by lumping the first passage time distribution to set the states (lumped) of a Markov chain, lumpability theory of Markov chain is required. Therefore, lumpability properties of Markov chains are described in this section

The theory of lumpability provides us with a powerful tool dealing with preserving the Markov property. Simply speaking, under certain conditions, if the aggregated chain still has the Markov property, we say that the original chain is lumpable.

In sense of preserving the Markov property, the simplest lumping will be lumping all the states to one single lump, that way, we will be able to have preserved the Markov property perfectly. But for single state chains, we can not observe any transition so we can not study the chain.

We provide a simple natural guidance on how to lump.

Consider a discrete parameter finite state irreducible Markov Chain $X=\left\{X_{t}: t=1,2, \ldots.\right\}$ with finite state space $S=\{1,2, \ldots, n\}$, set of all possible initial probability row vectors

$\mathrm{A}=\left\{\alpha \in \mathfrak{R}^{n} \mid \alpha .=\left(\alpha_{1}, \alpha_{2}, \ldots . ., \alpha_{n}\right), \sum_{i=1}^{n} \alpha_{i}=1, \alpha_{i} \geq 0, i=1, \ldots \ldots ., n\right\}$ Let $\zeta=\{C(1), C(2), \ldots \ldots, C(m)\}$ be a nontrivial mutually exclusive and exhaustive partition of $\mathrm{S}$, i.e. $m<n, \bigcup_{i=1}^{m} C(i)=S$ and $C(i) \cap C(j)=\phi$ for any $i \neq j$. The corresponding state space is $\bar{S}=\{1,2, \ldots \ldots \ldots, m\}$.We define the aggregated chain $\widetilde{X}=\left\{\widetilde{X}_{t}: t=1,2, \ldots.\right\}$ with state space $\bar{S}$ of the original chain $\mathrm{X}$ and the partiton $\zeta$ as follows [14].

$$
\tilde{X}_{t}=i \text { if and only if } X_{t} \in C(i) \text {, for } \mathrm{t}=0,1, . .
$$

Definition 2.2.1: If $\tilde{X}$ is Markovian for any initial probability vector $\alpha$ and the transition probabilities do not depend on the choise of $\alpha$, then we say $\mathrm{X}$ is strongly lumpable with respect to $\zeta$

If $\tilde{X}$ is Markovian for at least one initial probability vector but not all of them, then we say $\mathrm{X}$ is 
weakly lumpable with respect to $\zeta$

The fundamental conditions defining strong lumpability are given by Kemeny and Snell [15] in Theorems 2.2.1 and 2.2.2.

Theorem 2.2.1: A necessary and sufficient condition for a Markov chain to be strongly lumpable with recpect to a partition $\zeta=\{C(1), C(2), \ldots \ldots, C(m)\}$ is that for every pair of sets $C(i)$ and $C(j)$, the probabilities

$$
\tilde{P}_{i j}=\sum_{r \in C(j)} P_{k r}, i, j=1,2, \ldots \ldots, m
$$

for all $k \in C(i)$.

A well-known characterization of strong lumpability is also given by Kemeny and Snell .

Theorem 2.2.2: $\mathrm{X}$ is strongly lumpable to $\tilde{X}$ if and only if there exist matrices $U$ and $V$ such that

$$
V U P V=P V
$$

Where $\mathrm{V}$ is an $\mathrm{n} \times \mathrm{m}$ matrix whose jth column is a vector with ones in the components corresponding to states in $C(\mathrm{j})$ and zeros otherwise, and $U$ is an $\mathrm{m} x \mathrm{x}$ matrix whose ith row is a probability vector with nonzero components for states in $\mathrm{C}(\mathrm{i})$ and 0 for remaining states.

$\mathrm{V}$ is usually called the collector ( or aggregator). Its n-dimensional column vectors are orthogonal;

$\mathrm{U}$ is usually called a distributor and can be thought as $V^{T}$ with rows normalized to probability vectors (one way to define $\mathrm{U}$ is $U=\left(V^{T} V\right)^{-1} V^{T}$ ) or can be obtained from the steady state probabilities and V.

The transition probability matrix $\tilde{\mathrm{P}}$ of $\hat{X}$ is $\widetilde{\mathrm{P}}=\mathrm{UPV}$. It is easy to see that $\mathrm{UV}=\mathrm{I}$. And the k-step transiton matrix of the lumped chain can be obtained as follows:

$$
(\widetilde{P})^{k}=(U P V)^{k}=U P{ }^{k} V=\widetilde{P}^{k}
$$

Theorem 2.2.3: If $\tilde{\mathrm{P}}$ is the transition probability matrix of the aggregated homogeneous Markov chain $\tilde{X}=(\alpha, P, \zeta)$ then $\tilde{\mathrm{P}}$ is the same for every $\alpha$ leading to a Markov chain the unique transition probability matrix of $\tilde{X}$ is $\widetilde{P}=U_{\alpha} P V$
A useful sufficient condition to weak lumpability is also given by Kemeny and Snell next.

Theorem 2.2.4: $U_{\alpha} P V U_{\alpha}=U_{\alpha} P$ is a sufficient condition for $X$ to be weakly lumpable.

\section{Numerical Example and Results}

In this chapter, the first passage time distribution was determined by means of the lumping method based on Discrete First Passage Time algorithm and simulation of the Markov chain. It was understood upon the study carried out after application of the lumping method that the first passage time distributions varied depending on the form of lumping.

Discrete First Passage Time Distribution Algorithm

Step 1: Simulate Markov chain

Step 2: Choose starting state in state space

Step 3: Choose entering state in state space

Step 4: Find starting state and entering state orders in chain

Step 5: Calculate lengths between starting state and entering state if entering state order is greater than starting state order

Step 6: Calculate frequency of these lengths

The software "Easy Fit" was used to evaluate the first passage time distribution fitting. Based on the trial numbers of first passage time distribution, the best fitting distributions were obtained according to the results of Kolmogorov-Smirnov test on 65 distributions through the Easy Fit software. Table 2 -5 show the said distributions. In Table 3- 5 repersents $P_{K o l m}: \mathrm{p}$-value for Kolmogorov Smirnov test, and $T_{\text {Kolm }}$ : statistical value for Kolmogorov-Smirnov test.

We considered Markov chain with four-state to simplify calculation and save space.

Transition matrix has been used in all the of simulation studies made in this section

$$
P=\left[\begin{array}{llll}
0.40 & 0.22 & 0.18 & 0.20 \\
0.16 & 0.50 & 0.20 & 0.14 \\
0.28 & 0.32 & 0.30 & 0.10 \\
0.33 & 0.17 & 0.35 & 0.15
\end{array}\right]
$$

the distribution of first passage time was tried to be 
Table 1 Lumped states transition matrix according to the form of lumping.

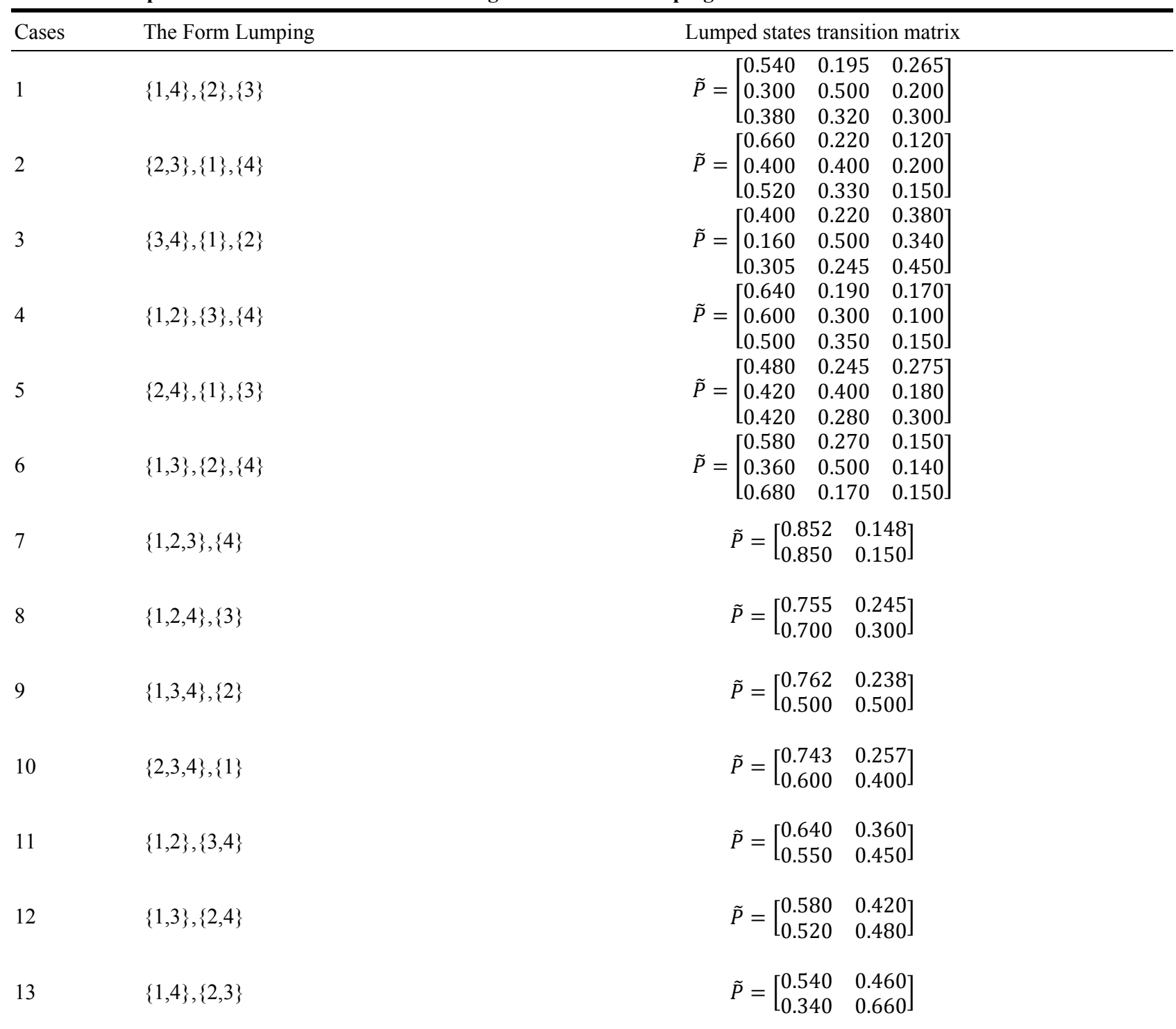

determined by generating $10.000,100.000,1.000 .000$ observations; respectively, with simulation from Markov chain in (3.1)

Lumped states transition matrices are used below to determine these distributions.

The simulation results regarding the first passage time distribution by lumping are given in the following table.

From Table 2, In determining the first passage time distribution, the form of lumping is considered to be quite effective. In other words, the first passage time distribution is also changing in accordance with the form of lumping.

According to the number of trial by lumping the first passage time distribution simulation results are given in the Table 3, Table 4, Table 5 .

Table 6 , Table 7 , Table 8 has been prepared to understand how the probability of the first passage time distributions based on a certain value when lumping in Markov chain has changed. From these tables, the first passage time probabilities are usually understood to be close to each other. These tables give information about the substitution of distributions when lumping in Markov. 
Table 2 The first passage time distribution obtained by lumping.

\begin{tabular}{|l|l|c|l|l|}
\hline Cases & $\begin{array}{l}\text { Lumping Form } \\
\text { and Lumped } \\
\text { States }\end{array}$ & $\begin{array}{l}\text { Starting } \\
\text { State }\end{array}$ & $\begin{array}{l}\text { Entering } \\
\text { Lumped State }\end{array}$ & $\begin{array}{l}\text { First Passage Time } \\
\text { Distribution }\end{array}$ \\
\hline 1 & $\{1,4\},\{2\},\{3\}$ & $\{2\}$ & $\{1,4\}$ & Weibull \\
\hline 3 & $\{2,3\},\{1\},\{4\}$ & $\{1\}$ & $\{2,3\}$ & Kumaraswamy \\
\hline 4 & $\{3,4\},\{1\},\{2\}$ & $\{1\}$ & $\{3,4\}$ & Johnson SB \\
\hline 5 & $\{2,4\},\{1\},\{3\}$ & $\{1\}$ & $\{2,4\}$ & Kumaraswamy \\
\hline 6 & $\{1,3\},\{2\},\{4\}$ & $\{2\}$ & $\{1,3\}$ & Weibull \\
\hline 7 & $\{1,2,3\},\{4\}$ & $\{4\}$ & $\{1,2,3\}$ & Burr(4P) \\
\hline 8 & $\{1,2,4\},\{3\}$ & $\{3\}$ & $\{1,2,4\}$ & Power Function \\
\hline 9 & $\{1,3,4\},\{2\}$ & $\{2\}$ & $\{1,3,4\}$ & Lognormal(3P) \\
\hline 10 & $\{2,3,4\},\{1\}$ & $\{1\}$ & $\{2,3,4\}$ & Power Function \\
\hline 11 & $\{1,2\},\{3,4\}$ & $\{3,4\}$ & $\{1,2\}$ & Gamma \\
\hline 12 & $\{1,2\},\{3,4\}$ & $\{1,2\}$ & $\{3,4\}$ & Weibull \\
\hline 13 & $\{1,3\},\{2,4\}$ & $\{2,4\}$ & $\{1,3\}$ & Lognormal(3P) \\
\hline 14 & $\{1,3\},\{2,4\}$ & $\{1,3\}$ & $\{2,4\}$ & Kumaraswamy \\
\hline 15 & $\{1,4\},\{2,3\}$ & $\{2,3\}$ & $\{1,4\}$ & Weibull \\
\hline 16 & $\{1,4\},\{2,3\}$ & $\{1,4\}$ & $\{2,3\}$ & Lognormal(3P) \\
\hline
\end{tabular}


Table 3 First passage time distributions when one lumping is made.

\begin{tabular}{|c|c|c|c|}
\hline \multirow{3}{*}{$\begin{array}{l}\text { Number of } \\
\text { Trial }\end{array}$} & \multicolumn{3}{|c|}{ Lumping Form and Lumped States } \\
\hline & $\{1,2\},\{3\},\{4\}$ & $\{1,3\},\{2\},\{4\}$ & $\{1,4\},\{2\},\{3\}$ \\
\hline & $\{3\} \rightarrow\{1,2\}$ & $\{2\} \rightarrow\{1,3\}$ & $\{2\} \rightarrow\{1,4\}$ \\
\hline \multirow{4}{*}{10.000} & $\begin{array}{l}\text { Power Function } \\
P_{\text {sin }}=0,54334 \\
T_{x=0}=0,23851\end{array}$ & $\begin{array}{l}\text { Weibull } \\
P_{\text {sim }}=0,40839 \\
T_{x+m}=0,24371\end{array}$ & $\begin{array}{l}\text { Weibull } \\
P_{\text {sin }}=0,54654 \\
T_{x+m}=0,17977\end{array}$ \\
\hline & $\begin{array}{l}\text { Gamma } \\
P_{\text {sim }}=0,30098 \\
T_{x=0}=0,29153\end{array}$ & $\begin{array}{l}\text { Gumbel Max } \\
P_{\text {sim }}=0,3947 \\
T_{x=0}=0,2465\end{array}$ & $\begin{array}{l}\text { Gen. Gamma } \\
P_{\sin }=0,50881 \\
T_{x+m}=0,18524\end{array}$ \\
\hline & $\begin{array}{l}\text { Inv.Gaussian } \\
P_{\text {sin }}=0,2821 \\
T_{x+m}=0,29671\end{array}$ & $\begin{array}{l}\text { Log-Logistic } \\
P_{\sin }=0,38971 \\
T_{x+\frac{1}{2}}=0,24753\end{array}$ & $\begin{array}{l}\text { Johnson SB } \\
P_{\text {sim }}=0,49261 \\
T_{\text {xom }}=0,18764 \\
\end{array}$ \\
\hline & $\begin{array}{l}\text { Gumbel Max } \\
P_{\text {sim }}=0,2785 \\
T_{x+m}=0,29772\end{array}$ & $\begin{array}{l}\text { Normal } \\
P_{\text {sim }}=0,36638 \\
T_{x+m}=0,25246\end{array}$ & $\begin{array}{l}\text { Gamma } \\
P_{\text {sim }}=0,37794 \\
T_{x+m}=0,20593\end{array}$ \\
\hline \multirow{4}{*}{100.000} & $\begin{array}{l}\text { PowerFunction } \\
P_{\text {xom }} \mathbf{- 0 , 3 0 3 5 8} \\
T_{\text {xom }}=0,26676\end{array}$ & $\begin{array}{l}\text { Weibull } \\
P_{\text {xom }}=0,23829 \\
T_{x+m}=0,24748\end{array}$ & $\begin{array}{l}\text { Weibull } \\
P_{\text {xom }}=0,33759 \\
T_{x+i n}=0,18196\end{array}$ \\
\hline & $\begin{array}{l}\text { Gamma } \\
P_{x+m}=0,24423 \\
T_{x+m}=0,28226\end{array}$ & $\begin{array}{l}\text { Gumbel Max } \\
P_{\text {xom }}=0,23495 \\
T_{x a+n}=0,24832\end{array}$ & $\begin{array}{l}\text { Johnson SB } \\
P_{x \min }=0,32006 \\
T_{x+2}=0,18478\end{array}$ \\
\hline & $\begin{array}{l}\text { Gumbel Max } \\
P_{x \cdot m}=0,20485 \\
T_{x+m}=0,29415\end{array}$ & $\begin{array}{l}\text { Normal } \\
P_{\text {xom }} \mathbf{- 0 , 2 1 2 9 2} \\
T_{\text {xan }}=0,25413\end{array}$ & $\begin{array}{l}\text { Gen. Gamma } \\
P_{x+m}=0,28224 \\
T_{x+n}=0,19125\end{array}$ \\
\hline & $\begin{array}{l}\text { Inv.Gsussian } \\
P_{\text {xom }} \mathbf{-} 0,19922 \\
T_{x+m}=0,29599\end{array}$ & $\begin{array}{l}\text { Johnson SB } \\
P_{x+m} \mathbf{~} 0,20842 \\
T_{x+m}=0,25537\end{array}$ & $\begin{array}{l}\text { Gamma } \\
P_{\text {Non }}=0,20057 \\
T_{x+m}=0,20773\end{array}$ \\
\hline \multirow{4}{*}{1.000 .000} & $\begin{array}{l}\text { Gamma } \\
P_{x+m}=0,15941 \\
T_{x=m}=0,27893\end{array}$ & $\begin{array}{l}\text { Weibull } \\
P_{\text {xom }}=0,16393 \\
T_{\text {xom }}=0,24154\end{array}$ & $\begin{array}{l}\text { Weibull } \\
P_{\text {xom }} \mathbf{- 0 , 2 4 1 5 3} \\
T_{\text {xom }}=0,18213\end{array}$ \\
\hline & $\begin{array}{l}\text { Gumbel Max } \\
P_{x \cdot m}=0,13041 \\
T_{x \cdot m}=0,28999\end{array}$ & $\begin{array}{l}\text { Kumaraswamy } \\
P_{\text {xom }}=0,15476 \\
T_{\text {xom }}=0,24436\end{array}$ & $\begin{array}{l}\text { Johnson SB } \\
P_{\text {x.m }}=0,24032 \\
T_{\text {Kalm }}=0,18235\end{array}$ \\
\hline & $\begin{array}{l}\text { Inv.Gaussian } \\
P_{\operatorname{sim}}-0,123 \\
T_{\sin }=0,29313\end{array}$ & $\begin{array}{l}\text { Gumbel Max } \\
P_{\text {xom }}=0,14894 \\
T_{\text {. }}=0,24622\end{array}$ & $\begin{array}{l}\text { Gen. Gamma } \\
P_{\text {xam }} \mathbf{- 0 , 2 0 4 8 5} \\
T_{\text {san }}=0,18927\end{array}$ \\
\hline & $\begin{array}{l}\text { Weibull } \\
P_{\text {xom }}=0,12121 \\
T_{x+m}=0,29391\end{array}$ & $\begin{array}{l}\text { Johnson SB } \\
P_{\operatorname{sim}}=0,1293 \\
T_{x+m}=0,25295\end{array}$ & $\begin{array}{l}\text { Gamma } \\
P_{\sin }=0,1316 \\
T_{x+m}=0,2072\end{array}$ \\
\hline
\end{tabular}

\begin{tabular}{|c|c|c|c|}
\hline \multirow{3}{*}{$\begin{array}{c}\text { Number of } \\
\text { Trial }\end{array}$} & \multicolumn{3}{|c|}{ Lumping Form and Lumped States } \\
\hline & $\{2,3\},\{1\},\{4\}$ & $\{2,4\},\{1\},\{3\}$ & $\{3,4\},\{1\},\{2\}$ \\
\hline & $\{1\} \rightarrow\{2,3\}$ & $\{1\} \rightarrow\{2,4\}$ & $\{1\} \rightarrow\{3,4\}$ \\
\hline \multirow{7}{*}{10.000} & $\begin{array}{l}\text { Gen. Gamma (4P) } \\
P_{\text {xam }}=0,49362 \\
T_{\text {san }}=0,21141\end{array}$ & $\begin{array}{l}\text { Weibull } \\
P_{\text {xem }}=0,34877 \\
T_{\operatorname{man}}=0,21697\end{array}$ & $\begin{array}{l}\text { JohnsonSB } \\
P_{\text {nex }}=0,83051 \\
T_{\operatorname{mat}}=0,11269\end{array}$ \\
\hline & $\begin{array}{l}\text { Weibull } \\
P_{x=-}=0,38837 \\
T=0.23027\end{array}$ & $\begin{array}{l}\text { Inv. Gaussian } \\
P_{\text {K*- }}=0,28502 \\
T=0,22071\end{array}$ & $\begin{array}{l}\text { Chi-Squared } \\
P_{\text {x*m }}=0,75903 \\
T=0,12142\end{array}$ \\
\hline & Log-Logistic & Lognormal (3P) & Beta \\
\hline & $P_{x=0}=0,34379$ & $P_{x k-}=0,26371$ & $P_{x \times-m}=0,75281$ \\
\hline & $T_{\operatorname{man}}=0,23914$ & $T_{\operatorname{san}}=0,23441$ & $T_{\operatorname{man}}=0,12214$ \\
\hline & $\begin{array}{l}\text { Inv.Gaussian } \\
P_{x *-1}=0,33766\end{array}$ & $\begin{array}{l}\text { Log-Logistic } \\
P_{x+m}=0,25754\end{array}$ & $\begin{array}{l}\text { Bufr } \\
P_{\text {Kt. }}=0,73683\end{array}$ \\
\hline & $T_{\operatorname{man}}=0,24041$ & $T_{\operatorname{man}}=0,23582$ & $T_{\operatorname{man}}=0,12398$ \\
\hline \multirow{5}{*}{100.000} & $\begin{array}{l}\text { Rumaraswany } \\
P_{\text {xw- }}=0,44909 \\
T_{\text {mam }}=0,19427\end{array}$ & $\begin{array}{l}\text { Kumaraswany } \\
P_{\text {xem }}-0,61987 \\
T_{\text {sam }}=0,16515\end{array}$ & $\begin{array}{l}\text { Johnson SB } \\
P_{\text {ne- }}=0,70173 \\
T_{\sin }=0,11187\end{array}$ \\
\hline & $\begin{array}{l}\text { Weibull } \\
P_{\text {xum }}=0,25366 \\
T_{\text {an }}=0,2303\end{array}$ & $\begin{array}{l}\text { Weibull(3P) } \\
P_{\text {nem }}=0,49979 \\
T_{\sin }=0,18179\end{array}$ & $\begin{array}{l}\text { Beta } \\
P_{\text {xe- }}-0,65859 \\
T_{\text {ax }}=0,11607\end{array}$ \\
\hline & $\begin{array}{l}\text { Gumbel Max } \\
P_{\text {xum }}=0,23107 \\
T_{\text {max }}=0,2356\end{array}$ & $\begin{array}{l}\text { Weibull } \\
P_{\text {xw- }}=0,31171 \\
T_{\text {man }}=0,2125\end{array}$ & $\begin{array}{l}\text { Bufr } \\
P_{\text {rex }}-0,58146 \\
T_{\text {max }}=0,12358\end{array}$ \\
\hline & $\begin{array}{l}\text { JohnsonSB } \\
P_{x=-}=0,20646\end{array}$ & $\begin{array}{l}\text { Gumbel Max } \\
P_{\text {x.m }}=0,23205\end{array}$ & $\begin{array}{l}\text { Weibull } \\
P_{\text {xem }}-0,55682\end{array}$ \\
\hline & $T_{\operatorname{man}}=0,24184$ & $T_{\operatorname{man}}=0,22932$ & $T_{\operatorname{man}}=0,12602$ \\
\hline \multirow{7}{*}{1.000 .000} & $\begin{array}{l}\text { Kumaraswany } \\
P_{\text {xam }}=0,34426 \\
T_{\text {am }}=0,19242\end{array}$ & $\begin{array}{l}\text { Kumaraswany } \\
P_{\text {xe- }}=0,40811 \\
T_{\sin }=0,17852\end{array}$ & $\begin{array}{l}\text { Johnson SB } \\
P_{\text {nex }}=0,55271 \\
T_{\max }=0,11258\end{array}$ \\
\hline & $\begin{array}{l}\text { Weibull } \\
P_{x=-}=0,18086\end{array}$ & $\begin{array}{l}\text { Weibull } \\
P_{x *-}=0,22776\end{array}$ & $\begin{array}{l}\text { Bum } \\
P_{\text {xe- }}-0,44773\end{array}$ \\
\hline & $T_{\max }=0,22601$ & $T_{\max }=0,21007$ & $T_{\operatorname{man}}=0,1223$ \\
\hline & $\begin{array}{l}\text { Gumbel Nax } \\
P_{x=m}=0,16043\end{array}$ & $\begin{array}{l}\text { Gumbel Max } \\
P_{x *-}=0,16601\end{array}$ & $\begin{array}{l}\text { Weibull } \\
P_{x \leqslant-}-0,41223\end{array}$ \\
\hline & $T_{\operatorname{man}}=0,23169$ & $T_{\operatorname{man}}=0,2252$ & $T_{\operatorname{man}}=0,12584$ \\
\hline & $\begin{array}{l}\text { JohnsonSB } \\
P_{x * m}=0,13534\end{array}$ & $\begin{array}{l}\text { JohnsonSB } \\
P_{\text {xem }}=0,15633\end{array}$ & $\begin{array}{l}\text { Chi-Squared } \\
P_{x=-}-0,3907\end{array}$ \\
\hline & $T_{\operatorname{man}}=0,23952$ & $T_{\operatorname{man}}=0,22795$ & $T_{\sin }=0,12807$ \\
\hline
\end{tabular}


Table 4 First passage time distributions when two lumpings are made.

\begin{tabular}{|c|c|c|c|c|c|c|}
\hline \multirow{3}{*}{$\begin{array}{l}\text { Number } \\
\text { of Trial }\end{array}$} & \multicolumn{6}{|c|}{ Lumping Form and Lumped States } \\
\hline & $\{1,2\},\{3,4\}$ & $\{1,2\},\{3,4\}$ & $\{1,3\},\{2,4\}$ & $\{1,3\},\{2,4\}$ & $\{1,4\},\{2,3\}$ & $\{1,4\},\{2,3\}$ \\
\hline & $\{3,4\} \rightarrow\{1,2\}$ & $\{1,2\} \rightarrow\{3,4\}$ & $\{2,4\} \rightarrow\{1,3\}$ & $\{1,3\} \rightarrow\{2,4\}$ & $\{2,3\} \rightarrow\{1,4\}$ & $\{1,4\} \rightarrow\{2,3\}$ \\
\hline \multirow{4}{*}{10.000} & $\begin{array}{l}\text { Inv. Gallssian } \\
P_{\text {xass }}=0,29937 \\
T_{\text {xaix }}=0,29197\end{array}$ & $\begin{array}{l}\text { Weibull } \\
P_{\text {xos }}=0,39011 \\
T_{x \operatorname{six}}=0,19379\end{array}$ & $\begin{array}{l}\text { Burr (4P) } \\
P_{\text {xas }}=0,37689 \\
T_{\text {xaix }}=0,26073\end{array}$ & $\begin{array}{l}\text { Weibull } \\
P_{\text {xas }}=0,43459 \\
T_{\text {xaix }}=0,22169\end{array}$ & $\begin{array}{l}\text { Gen. Gamma } \\
P_{x a s a}=0,52937 \\
T_{x a i x}=0,17758\end{array}$ & $\begin{array}{l}\text { Weibull } \\
P_{\text {xasx }}=0,37612 \\
T_{\text {xoin }}=0,24102\end{array}$ \\
\hline & $\begin{array}{l}\text { Gumbel Max } \\
P_{\text {xast }}=0,29683 \\
T_{\text {xeis }}=0,29265\end{array}$ & $\begin{array}{l}\text { Johnson SB } \\
P_{\text {xas }}=0,34737 \\
T_{\text {xoix }}=0,20092\end{array}$ & $\begin{array}{l}\text { Gumbel Max } \\
P_{\text {xasx }}=0,33406 \\
T_{\text {xaix }}=0,27054\end{array}$ & $\begin{array}{l}\text { Johnson SB } \\
P_{\text {xost }}=0,3855 \\
T_{\text {xoix }}=0,23082\end{array}$ & $\begin{array}{l}\text { Weibull } \\
P_{x_{0 x x}}=0,51017 \\
T_{x \text { oix }}=0,1803\end{array}$ & $\begin{array}{l}\text { Gumbel Max } \\
P_{\text {xas }}=0,35648 \\
T_{\text {xaix }}=0,24507\end{array}$ \\
\hline & $\begin{array}{l}\text { Gamma } \\
P_{\text {xass }}=0,28274 \\
T_{\text {xais }}=0,29653\end{array}$ & $\begin{array}{l}\text { Gen. Gamma } \\
P_{\text {xas }}=0,29615 \\
T_{\text {xaix }}=0,21026\end{array}$ & $\begin{array}{l}\text { Inv. Gaussian } \\
P_{\text {xase }}=0,30841 \\
T_{\text {xaix }}=0,27682\end{array}$ & $\begin{array}{l}\text { LogLogistic } \\
P_{\text {xast }}=0,35693 \\
T_{\text {xoix }}=0,23645\end{array}$ & $\begin{array}{l}\text { Johnson SB } \\
P_{\text {xass }}=0,46552 \\
T_{\text {xais }}=0,18682\end{array}$ & $\begin{array}{l}\text { Normal } \\
P_{x_{\text {os }}}=0,33193 \\
T_{\text {xais }}=0,25034\end{array}$ \\
\hline & $\begin{array}{l}\text { Weibull } \\
P_{\text {xast }}=0,2785 \\
T_{x \text { aix }}=0,29772\end{array}$ & $\begin{array}{l}\text { Gumbel Max } \\
P_{\text {xas }}=0,24064 \\
T_{\text {xoix }}=0,22177\end{array}$ & $\begin{array}{l}\text { Weibull } \\
P_{\text {xasx }}=0,30226 \\
T_{\text {xoix }}=0,27837\end{array}$ & $\begin{array}{l}\text { Gumbel Max } \\
P_{\text {xas }}=0,35505 \\
T_{\text {xais }}=0,23683\end{array}$ & $\begin{array}{l}\text { Inv. Gaussian } \\
P_{\text {xas }}=0,40292 \\
T_{\text {xais }}=0,19653\end{array}$ & $\begin{array}{l}\text { Johnson SB } \\
P_{\text {xosx }}=0,31939 \\
T_{\text {xast }}=0,25313\end{array}$ \\
\hline \multirow{4}{*}{100.000} & $\begin{array}{l}\text { Power Function } \\
P_{\text {xast }}=0,32983 \\
T_{\text {xalu }}=0,26057\end{array}$ & $\begin{array}{l}\text { Weibull } \\
P_{\text {xast }}=0,36492 \\
T_{\text {xoix }}=0,18905\end{array}$ & $\begin{array}{l}\text { Lognormal (3P) } \\
P_{x_{0} \text { sx }}=0,64589 \\
T_{\text {xaix }}=0,19369\end{array}$ & $\begin{array}{l}\text { Weibull } \\
P_{\text {xost }}=0,30807 \\
T_{\text {xais }}=0,2132\end{array}$ & $\begin{array}{l}\text { Weibull } \\
P_{\text {xost }}=0,36356 \\
T_{\text {xoix }}=0,17795\end{array}$ & $\begin{array}{l}\text { Lognormal(3P) } \\
P_{\text {xost }}=0,35807 \\
T_{\text {xaix }}=0,21524\end{array}$ \\
\hline & $\begin{array}{l}\text { Gamma } \\
P_{\text {xast }}=0,22913 \\
T_{\text {xais }}=0,28664\end{array}$ & $\begin{array}{l}\text { Johnson SB } \\
P_{\text {yos }}=0,32695 \\
T_{\text {xoix }}=0,19535\end{array}$ & $\begin{array}{l}\text { Gen.Gamma }(4 \mathrm{P}) \\
P_{\text {xas }}=0,29962 \\
T_{\text {xaix }}=0,25768\end{array}$ & $\begin{array}{l}\text { Gumbel Max } \\
P_{\text {xas }}=0,23911 \\
T_{\text {xain }}=0,22767\end{array}$ & $\begin{array}{l}\text { Johnson SB } \\
P_{\text {xost }}=0,33961 \\
T_{\text {xais }}=0,18164\end{array}$ & $\begin{array}{l}\text { Weibull } \\
P_{\text {yost }}=0,25928 \\
T_{\text {xoix }}=0,23542\end{array}$ \\
\hline & $\begin{array}{l}\text { Gumbel Max } \\
P_{\text {xoss }}=0,22345 \\
T_{\text {xais }}=0,28834\end{array}$ & $\begin{array}{l}\text { Gen.Gamma } \\
P_{\text {xas }}=0,29781 \\
T_{\text {xaix }}=0,20052\end{array}$ & $\begin{array}{l}\text { Gumbel Max } \\
P_{\text {xos }}=0,26352 \\
T_{\text {xaix }}=0,26657\end{array}$ & $\begin{array}{l}\text { Johnson SB } \\
P_{\text {xoss }}=0,23248 \\
T_{\text {xois }}=0,22922\end{array}$ & $\begin{array}{l}\text { Gen. Gamma } \\
P_{\text {xass }}=0,30017 \\
T_{\text {xas }}=0,18811\end{array}$ & $\begin{array}{l}\text { Gumbel Max } \\
P_{\text {yost }}=0,2509 \\
T_{\text {xaix }}=0,23736\end{array}$ \\
\hline & $\begin{array}{l}\text { Inv.Gaussian } \\
P_{\text {xast }}=0,21455 \\
T_{\text {xais }}=0,29107\end{array}$ & $\begin{array}{l}\text { Gumbel Max } \\
P_{\text {xas }}=0,23384 \\
T_{\text {xaix }}=0,21328\end{array}$ & $\begin{array}{l}\text { Weibull } \\
P_{\text {xosx }}=0,22914 \\
T_{\text {xaix }}=0,27589\end{array}$ & $\begin{array}{l}\text { Inv.Gaussian } \\
P_{\text {xast }}=0,20161 \\
T_{\text {xaix }}=0,23689\end{array}$ & $\begin{array}{l}\text { Gamma } \\
P_{\text {xasa }}=0,2233 \\
T_{\text {xais }}=0,20271\end{array}$ & $\begin{array}{l}\text { Gamma } \\
P_{\text {xas }}=0,20088 \\
T_{\text {xais }}=0,25011\end{array}$ \\
\hline \multirow{4}{*}{1.000 .000} & $\begin{array}{l}\text { Gamma } \\
P_{\text {xais }}=0,10789 \\
T_{x \text { aix }}=0,28256\end{array}$ & $\begin{array}{l}\text { Weibull } \\
P_{\text {xast }}=0,27889 \\
T_{\text {xaix }}=0,18484\end{array}$ & $\begin{array}{l}\text { Lognormal (3P) } \\
P_{\text {xasx }}=0,40568 \\
T_{\text {xaix }}=0,20684\end{array}$ & $\begin{array}{l}\text { Kumaras wamy } \\
P_{\text {xast }}=0,45947 \\
T_{\text {xais }}=0,17892\end{array}$ & $\begin{array}{l}\text { Weibull } \\
P_{\text {xast }}=0,30641 \\
T_{\text {xais }}=0,17707\end{array}$ & $\begin{array}{l}\text { Lognormal(3P) } \\
P_{\text {xost }}=0,30503 \\
T_{x_{\text {oin }}}=0,21378\end{array}$ \\
\hline & $\begin{array}{l}\text { Gumbel Max } \\
P_{\text {xas }}=0,10361 \\
T_{\text {xoin }}=0,28454\end{array}$ & $\begin{array}{l}\text { Johnson SB } \\
P_{\text {xosx }}=0,23056 \\
T_{\text {xoix }}=0,19383\end{array}$ & $\begin{array}{l}\text { Gen.Gamma }(4 \mathrm{P}) \\
P_{\text {xas }}=0,16814 \\
T_{\text {xaix }}=0,25983\end{array}$ & $\begin{array}{l}\text { Weibull } \\
P_{\text {xast }}=0,26628 \\
T_{\text {xoix }}=0,21122\end{array}$ & $\begin{array}{l}\text { Johnson SB } \\
P_{\text {yous }}=0,27899 \\
T_{\text {xoin }}=0,1816\end{array}$ & $\begin{array}{l}\text { Weibull } \\
P_{\text {xos }}=0,20956 \\
T_{x \operatorname{lix}}=0,23483\end{array}$ \\
\hline & $\begin{array}{l}\text { Inv.Gaussian } \\
P_{\text {xass }}=0,09501 \\
T_{\text {xain }}=0,28873\end{array}$ & $\begin{array}{l}\text { Gen. Gamma } \\
P_{x a s}=0,21172 \\
T_{x \text { oix }}=0,19771\end{array}$ & $\begin{array}{l}\text { Gumbel Max } \\
P_{\text {yosx }}=0,15502 \\
T_{\text {xaix }}=0,26414\end{array}$ & $\begin{array}{l}\text { Gumbel Max } \\
P_{\text {xase }}=0,20396 \\
T_{\text {xaix }}=0,22515\end{array}$ & $\begin{array}{l}\text { Gen. Gamma } \\
P_{\text {xosa }}=0,26951 \\
T_{x \text { aix }}=0,18323\end{array}$ & $\begin{array}{l}\text { Gumbel Max } \\
P_{\text {yas }}=0,20086 \\
T_{\text {xaix }}=0,23708\end{array}$ \\
\hline & $\begin{array}{l}\text { Weibull } \\
P_{\text {xast }}=0,09044 \\
T_{\text {xalu }}=0,29109\end{array}$ & $\begin{array}{l}\text { Kumaras wamy } \\
P_{\text {xas }}=0,20159 \\
T_{\text {xaix }}=0,19991\end{array}$ & $\begin{array}{l}\text { Gamma } \\
P_{x_{\text {a is }}}=0,13661 \\
T_{\text {xaix }}=0,27072\end{array}$ & $\begin{array}{l}\text { Johnson SB } \\
P_{\text {xost }}=0,18966 \\
T_{\text {xaix }}=0,22879\end{array}$ & $\begin{array}{l}\text { Gamma } \\
P_{\text {xas }}=0,18253 \\
T_{x a i x}=0,20072\end{array}$ & $\begin{array}{l}\text { Gamma } \\
P_{\text {xas }}=0,15677 \\
T_{\text {xais }}=0,24983\end{array}$ \\
\hline
\end{tabular}


The Form Lumping $\{2,4\},\{1\},\{3\}$

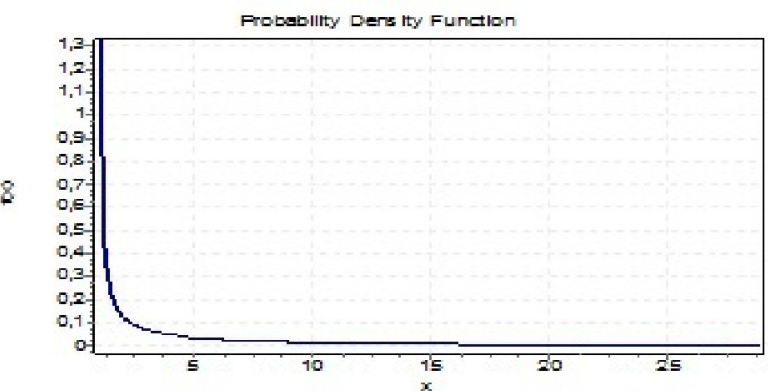

- Rumarawamy $(0,27072 ; 1,5758 ; 1,0 ; 78,874)$

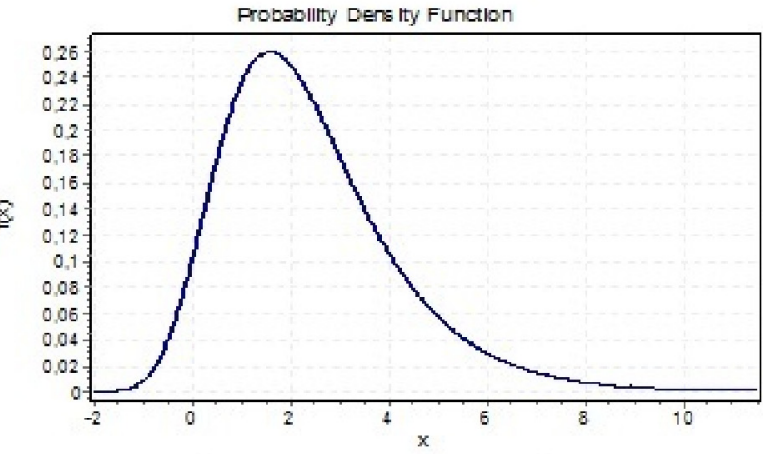

- GumbeIMax $(1,4164 ; 1,5656)$
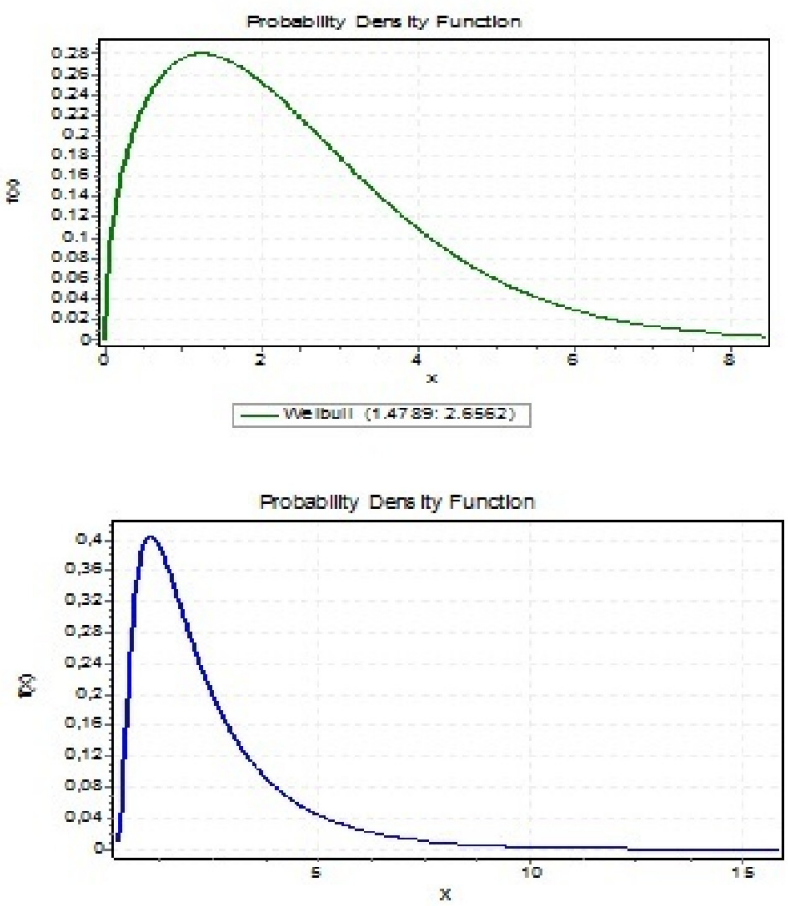

- Johnsen $89[3,1378 ; 1,1491 ; 27,007 ; 0,19951)$

Fig. 1 First passage time distribution graphs according to $\{2,4\},\{1\},\{3\}$ lumped states

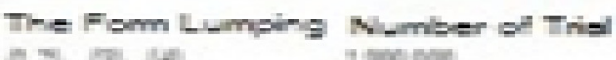

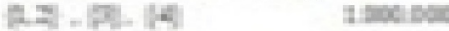

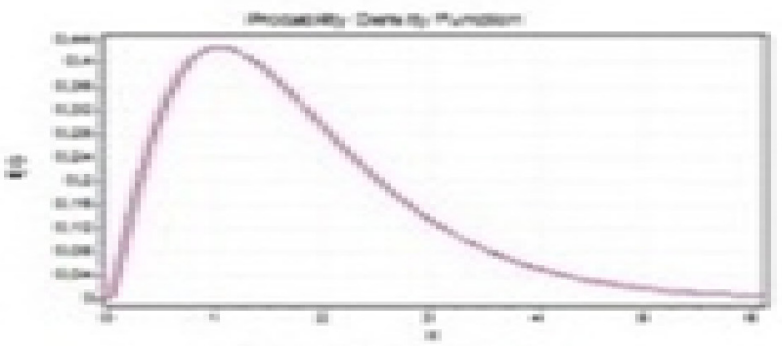

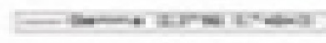

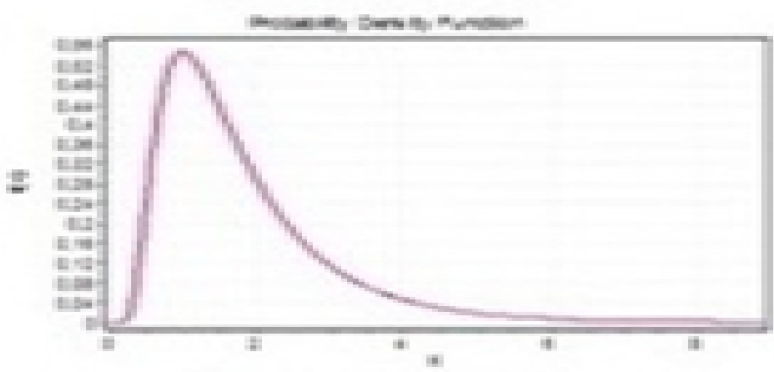


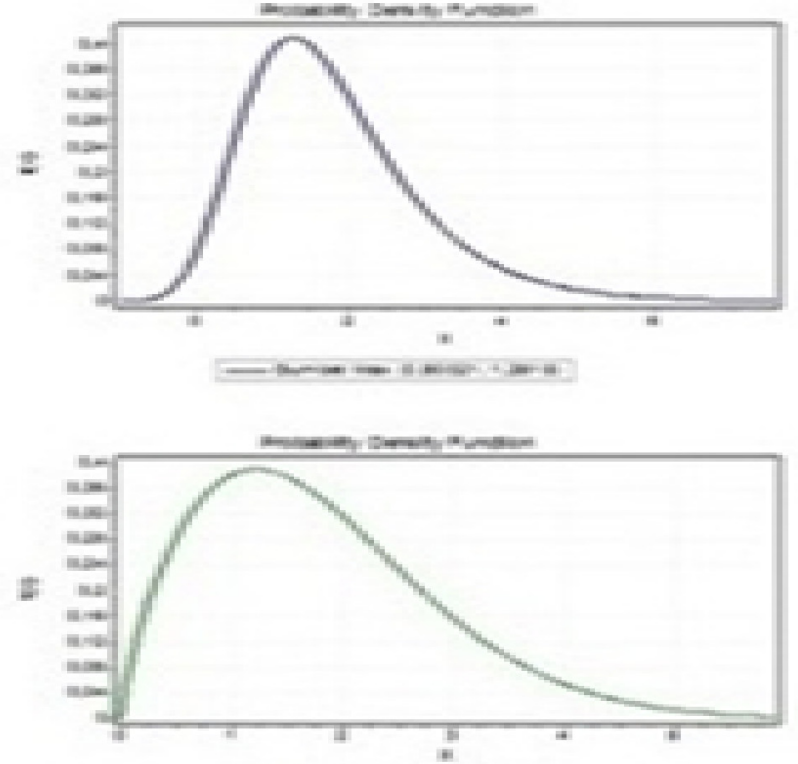

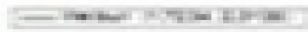

Fig. 2 First passage time distribution graphs according to $\{1,2\},\{3\},\{4\}$ lumped states. 
Table 5 First passage time distributions when one lump has three states.

\begin{tabular}{|c|c|c|c|c|}
\hline \multirow{3}{*}{$\begin{array}{l}\text { Number } \\
\text { of Trial }\end{array}$} & \multicolumn{4}{|c|}{ Lumping Form and Lumped States } \\
\hline & $\{1,2,3\},\{4\}$ & $\{1,2,4\},\{3\}$ & $\{1,3,4\},\{2\}$ & $\{2,3,4\},\{1\}$ \\
\hline & $\{4\} \rightarrow\{1,2,3\}$ & $\{3\} \rightarrow\{1,24\}$ & $\{2\} \rightarrow\{1,3,4\}$ & $\{1\} \rightarrow\{2,3,4\}$ \\
\hline \multirow{3}{*}{10.000} & 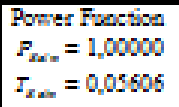 & $\begin{array}{l}\text { Logoormal (3P) } \\
P_{x<z}=0,9988 \\
I_{x-z}=0,12905\end{array}$ & $\begin{array}{l}\text { Logoorma1 (3P) } \\
P_{x=z}=0,61361 \\
I_{x z}=0,2154\end{array}$ & $\begin{array}{l}\text { Porrer Fraction } \\
P_{x x=}=0,31148 \\
I_{x a z}=0,19722\end{array}$ \\
\hline & 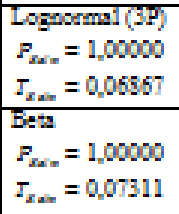 & 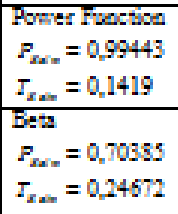 & 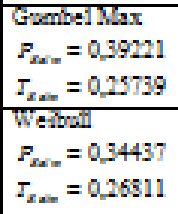 & 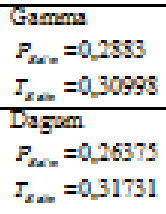 \\
\hline & 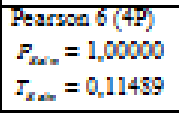 & $\begin{array}{l}\text { Dagsen } \\
P_{\text {sts }}=0,25492 \\
I_{x-z}=0,36045\end{array}$ & $\begin{array}{l}\text { Normal } \\
P_{x s z}=0,34045 \\
I_{x-z}=0,26903\end{array}$ & 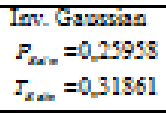 \\
\hline \multirow{4}{*}{100.000} & 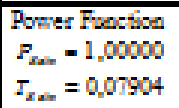 & 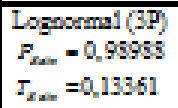 & $\begin{array}{l}\text { Grembel Max } \\
P_{\text {soz }}=0,26999 \\
I_{s \alpha z}=0,25569\end{array}$ &  \\
\hline & $\begin{array}{l}\text { Beta } \\
P_{\text {sta }}=0,99995 \\
I_{x \Delta}=0,11525\end{array}$ & 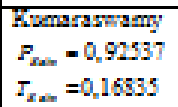 & $\begin{array}{l}\text { Weiball } \\
P_{\text {sex }}=0,2423 \\
I_{x \text { s. }}=0,26274\end{array}$ & 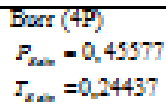 \\
\hline & 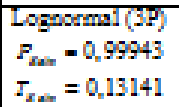 & $\begin{array}{l}\text { Ponter Foortion } \\
P_{\text {sti }}=0,9195 \\
I_{x-2}=0,17023\end{array}$ & 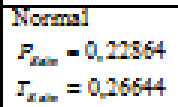 & $\begin{array}{l}\text { Gamma } \\
P_{x-2 .}=0,20692 \\
I_{x+2}=0,30592\end{array}$ \\
\hline & $\begin{array}{l}\text { Pearsod 6(4P) } \\
P_{\text {sai }}=0,99902 \\
T_{x a-}=0,13600\end{array}$ & $\begin{array}{l}\text { Beta } \\
P_{\text {xat }}=0,50745 \\
I_{x a-}=0,25902\end{array}$ & $\begin{array}{l}\text { Gamma } \\
P_{\text {sat }}=0,21483 \\
T_{x a}=0,27035\end{array}$ & 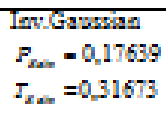 \\
\hline \multirow{4}{*}{1.000 .000} & $\begin{array}{l}\operatorname{Bxar}(4 \mathrm{P}) \\
P_{\text {sox }}=0,99599 \\
I_{x=2}=0,13966\end{array}$ & $\begin{array}{l}\text { Poncer Fuction } \\
P_{\text {xat }}=0,72013 \\
I_{x \leq-}=0,19657\end{array}$ & $\begin{array}{l}\text { LogoormaI(3P) } \\
P_{x=0}=0,28311 \\
I_{x a}=0,22399\end{array}$ & 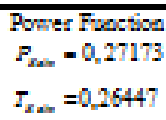 \\
\hline & $\begin{array}{l}\text { Logoormal (JP) } \\
P_{\text {sat }}=0,99011 \\
I_{x-2}=0,14904\end{array}$ &  & $\begin{array}{l}\text { GrembelMax } \\
P_{\text {sat }}=0,17471 \\
I_{x-2}=0,25078\end{array}$ & $\begin{array}{l}\text { Gamma } \\
P_{x a t}=0,15734 \\
I_{x a z}=0,29948\end{array}$ \\
\hline & $\begin{array}{l}\text { Rayleigh } \\
P_{\text {sat }}=0,10314 \\
I_{x a z}=0,43379\end{array}$ & 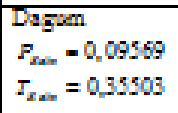 & $\begin{array}{l}\text { Normal } \\
P_{x \leq x}=0,14749 \\
I_{x a z}=0,25953\end{array}$ & 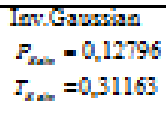 \\
\hline & 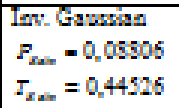 & 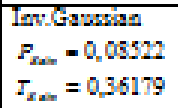 & 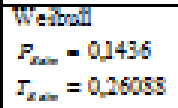 & $\begin{array}{l}\text { Grabe1Max } \\
P_{x-2}=0,1169 \\
T_{x-2}=0,31679\end{array}$ \\
\hline
\end{tabular}


Table 6 Compare first passage probabilities when one lump has two states.

\begin{tabular}{|c|c|c|c|c|c|}
\hline \multirow{2}{*}{$\begin{array}{l}\text { Lumping } \\
\text { Form and } \\
\text { Lumped States }\end{array}$} & \multirow{2}{*}{$\begin{array}{l}\text { Probability } \\
\text { Distribution }\end{array}$} & \multicolumn{4}{|c|}{1.000 .000 Trial } \\
\hline & & $P(X<2)$ & $P(X<5)$ & $P(2<X<4)$ & $P(X>6)$ \\
\hline $\multirow{4}{*}{1,2},\{3\}=\{4\}$ & Gamma & 0,65473 & 0,98287 & 0,29513 & 0,00561 \\
\hline & Gumbel Max & 0,64386 & 0,98440 & 0,30952 & 0,00516 \\
\hline & Inv.Gaussian & 0,68759 & 0,97854 & 0,26123 & 0,00920 \\
\hline & Weibull & 0,62786 & 0,99173 & 0,33391 & 0,00141 \\
\hline $\multirow{4}{*}{13},\{2\},\{4\}$ & Weibull & 0,52359 & 0,94481 & 0,35133 & 0,02239 \\
\hline & Kumaraswamy & 0,71828 & 0,86585 & 0,11738 & 0,11134 \\
\hline & Gumbel Max & 0,52278 & 0,93774 & 0,34753 & 0,02931 \\
\hline & Johnson SB & 0,59734 & 0,93283 & 0,28438 & 0,03872 \\
\hline $\multirow{4}{*}{14},\{2\} .\{3\}$ & Weibull & 0,40067 & 0,82817 & 0,32777 & 0,10518 \\
\hline & Johnson SB & 0,44183 & 0,84178 & 0,32000 & 0,10634 \\
\hline & Gen.Gamma & 0,38513 & 0,82809 & 0,34250 & 0,10598 \\
\hline & Gamma & 0,43436 & 0,82707 & 0,30466 & 0,11345 \\
\hline $\multirow{4}{*}{, 3},\{1\},\{4\}$ & Kumaraswamy & 0,55155 & 0,75287 & 0,15691 & 0,21221 \\
\hline & Weibull & 0,50173 & 0,93760 & 0,36040 & 0,02593 \\
\hline & Gumbel Max & 0,50256 & 0,93084 & 0,35616 & 0,03316 \\
\hline & Johnson SB & 0,56705 & 0,92801 & 0,30506 & 0,04115 \\
\hline $\multirow{4}{*}{2,4},\{1\},\{3\}$ & Kumaraswamy & 0,56004 & 0,75565 & 0,15262 & 0,21059 \\
\hline & Weibull & 0,48174 & 0,92179 & 0,35818 & 0,03554 \\
\hline & Gumbel Max & 0,47909 & 0,91530 & 0,35677 & 0,04275 \\
\hline & Johnson SB & 0,54189 & 0,91585 & 0,31323 & 0,04967 \\
\hline $\multirow{4}{*}{3,4},\{1\},\{2\}$ & Johnson SB & 0,28438 & 0,67361 & 0,28923 & 0,25029 \\
\hline & Burr & 0,27002 & 0,69897 & 0,32304 & 0,22546 \\
\hline & Weibull & 0,27545 & 0,64035 & 0,26242 & 0,27615 \\
\hline & Chi-Squared & 0,26424 & 0,71270 & 0,32975 & 0,19915 \\
\hline
\end{tabular}


Table 7 Compare first passage probabilities when each lump has two states.

\begin{tabular}{|c|c|c|c|c|c|}
\hline \multirow{2}{*}{$\begin{array}{l}\text { Lumping Form } \\
\text { And Lumped } \\
\text { States }\end{array}$} & \multirow{2}{*}{$\begin{array}{l}\text { Probability } \\
\text { Distribution }\end{array}$} & \multicolumn{4}{|c|}{1.000 .000 Trial } \\
\hline & & $P(X<2)$ & $P(X<5)$ & $P(2<X<4)$ & $P(X>6)$ \\
\hline \multirow{4}{*}{$\begin{array}{l}\{1,2\} \cdot\{3,4\} \\
\text { First Lump }\end{array}$} & Gamma & 0,64329 & 0,97788 & 0,29719 & 0,00793 \\
\hline & Gumbel Max & 0,62844 & 0,98025 & 0,31620 & 0,00696 \\
\hline & Inv.Gaussian & 0,67703 & 0,97387 & 0,26412 & 0,01187 \\
\hline & Weibull & 0,61287 & 0,98720 & 0,33767 & 0,00273 \\
\hline \multirow{4}{*}{$\begin{array}{l}\{1,2\},\{3,4\} \\
\text { Second Lump }\end{array}$} & Weibull & 0,41849 & 0,86036 & 0,34412 & 0,07851 \\
\hline & Johnson SB & 0,46960 & 0,86979 & 0,32531 & 0,08376 \\
\hline & Gen.Gamma & 0,41120 & 0,86421 & 0,35744 & 0,07743 \\
\hline & Kumaraswamy & 0,55998 & 0,74792 & 0,14673 & 0,21972 \\
\hline \multirow{4}{*}{$\begin{array}{l}\{1,3\},\{2,4\} \\
\text { First Lump }\end{array}$} & Lognomal(3P) & 0,72558 & 0,78695 & 0,04934 & 0,20397 \\
\hline & Gen. Gamma 4P & 0,77857 & 0,93175 & 0,12811 & 0,05181 \\
\hline & Gumbel Max & 0,59291 & 0,97126 & 0,33362 & 0,01108 \\
\hline & Gamma & 0,61299 & 0,96780 & 0,30913 & 0,01291 \\
\hline \multirow{4}{*}{$\begin{array}{l}\{1,3\},\{2,4\} \\
\text { Second Lump }\end{array}$} & Kumaraswamy & 0,56004 & 0,75565 & 0,15262 & 0,21059 \\
\hline & Weibull & 0,48305 & 0,92194 & 0,35731 & 0,03552 \\
\hline & Gumbel Max & 0,48004 & 0,91620 & 0,35707 & 0,04216 \\
\hline & Johnson SB & 0,54671 & 0,91553 & 0,30874 & 0,05005 \\
\hline \multirow{4}{*}{$\begin{array}{l}\{1,4\},\{2,3\} \\
\text { First Lump }\end{array}$} & Weibull & 0,39857 & 0,83573 & 0,33603 & 0,09784 \\
\hline & Johnson SB & 0,44158 & 0,84893 & 0,32707 & 0,09973 \\
\hline & Gen. Gamma & 0,39110 & 0,83973 & 0,34906 & 0,09639 \\
\hline & Gamma & 0,43332 & 0,83536 & 0,31314 & 0,10565 \\
\hline \multirow{4}{*}{$\begin{array}{l}\{1,4\},\{2,3\} \\
\text { Second Lump }\end{array}$} & Lognomal(3P) & 0,66752 & 0,75068 & 0,06686 & 0,23705 \\
\hline & Weibull & 0,52261 & 0,95006 & 0,35872 & 0,01908 \\
\hline & Gumbel Max & 0,52533 & 0,94404 & 0,35386 & 0,02542 \\
\hline & Gamma & 0,55534 & 0,93915 & 0,32183 & 0,02945 \\
\hline
\end{tabular}


Table 8 Compare first passage probabilities when one lump has three states

\begin{tabular}{|c|c|c|c|c|c|}
\hline \multirow{2}{*}{$\begin{array}{l}\text { Lumping } \\
\text { Form and } \\
\text { Lumped States }\end{array}$} & \multirow{2}{*}{$\begin{array}{l}\text { Probability } \\
\text { Distribution }\end{array}$} & \multicolumn{4}{|c|}{1.000 .000 Trial } \\
\hline & & $P(X<1,5)$ & $P(X<3)$ & $R(1,<x<105)$ & $P(X>2)$ \\
\hline $\multirow{4}{*}{1,2,3},\{4\}$ & Burr (4P) & 0,97815 & 0,99511 & 0,30604 & 0,01043 \\
\hline & Lognormal (3P) & 0,99988 & 0,99998 & 0,00382 & $5,5683 \mathrm{E}-5$ \\
\hline & Rayleigh & 0,72190 & 0,99402 & 0,02563 & 0,10278 \\
\hline & Inv. Gaussian & 0,79747 & 0,99694 & 0,04045 & 0,05433 \\
\hline $\multirow{4}{*}{1,2,4},\{3\}$ & Power Function & 0,87092 & 0,92314 & 0,05169 & 0,10335 \\
\hline & Johnson SB & 0,69592 & 0,94824 & 0,04120 & 0,15974 \\
\hline & Dagum & 0,87460 & 0,99597 & 0,07141 & 0,03086 \\
\hline & Inv. Gaussian & 0,63727 & 0,95367 & 0,02777 & 0,18543 \\
\hline $\multirow{4}{*}{1,3,4},\{2\}$ & Lognormal (3P) & 0,64626 & 0,71783 & 0,09269 & 0,31710 \\
\hline & Gumbel Max & 0,41205 & 0,79702 & 0,01269 & 0,43044 \\
\hline & Normal & 0,36084 & 0,76006 & 0,00892 & 0,50079 \\
\hline & Weibull & 0,40440 & 0,79192 & 0,01328 & 0,44005 \\
\hline $\multirow{4}{*}{2,3,4},\{1\}$ & Power Function & 0,83260 & 0,89475 & 0,05923 & 0,13689 \\
\hline & Gamma & 0,51912 & 0,89043 & 0,01846 & 0,30712 \\
\hline & Inv. Gaussian & 0,54715 & 0,89847 & 0,02317 & 0,27625 \\
\hline & Gumbel Max & 0,50173 & 0,89456 & 0,01750 & 0,31314 \\
\hline
\end{tabular}




\section{Conclusions}

Though the Markov chains involve a wide variety of applications, there is a limited number of studies to investigate the first passage time characteristics. Indeed, the first passage times constitute an important part of the Markov analysis.

As the first passage times have discrete for a state space finite irreducible Markov chain, this study involves a continuous distribution approach to discrete distribution. The Markov chain was simulated to demonstrate the first passage time distribution depending on the number of trials.

In this study is described the lumping method based on gathering the states with the aim of reducing the state space of the Markov chain in order to examine the complex systems in an easier manner. The first passage time distribution of a Markov chain into the lumped states was determined by means of lumping.

Table 3-5 show the distributions that allow the best fit on the basis of the results of Kolmogorov-Smirnov test among 65 distributions through the East Fit software, depending on the number of trials of the first passage time distribution.

It was tried to determine the first passage time distribution by means of the lumping into the irreducible Markov chain with four states discussed in the implementation chapter. Representing the first passage time distribution fitting, the p-value was minimum 0.08522 , where the first passage of the Markov chain from 3 states $\{1,2,4\}$ into the lumped states resulted in the Inv. Gaussian distribution.

Representing the first passage time distribution fitting, the p-value was maximum 1.000, where the first passage of the Markov chain from 4 states $\{1,2,3\}$ into the lumped states resulted in the Power Function distribution. It is observed in Table 2 that 16 possible first time distributions existed in an irreducible Markov chain with four states, where a better fitting was ensured in several distributions, including 4 Weibull, 3 Kumaraswamy, 3 Lognormal
(3P), 2 Gamma, 2 Power Function, 1 Johnson SB, and 1 Burr (4P) ones.

When lumping was used, the first passage time distribution varied depending on the lumping form as shown in Table 2. Furthermore, the first passage time distribution graphs were given. The probability distribution graphs for the lumped states shown in Fig. 1 and Fig. 2 demonstrate that the first passage time distribution is of the positive skew or reverse $\mathrm{j}$ shaped.

Table $6-8$ show the comparison of the probabilities based on a certain value upon application of the lumping method. It is understood through these tables that the first passage probabilities are usually close to each other.

It is possible for the future studies to adapt the first passage times in association with the lumping method into the Markov Decision processes. It would make it possible to use the lumping method to develop more flexible strategies by unifying the desired/adverse states.

To increase the fitting of data into the distributions, the generalized cases of the distributions are taken into account. It is very interesting to observe in studies carried out in the recent years by Alzaatreh et al. [16] that the transformations are used to generate new continuous distributions and new discrete distributions from the continuous distributions.

Alzaatreh et al.obtained new continuous and discrete distributions in the form of T-X distribution family. Being the T-X distribution family generated in an effort to generalize the distributions, the Beta-Gamma, Beta-Exponential,Exponential-Geometrical distributions could be examined for their fitting of the first passage time distributions, whereby the new distributions would likely have a better fitting than the existing distributions.

\section{References}

[1] A. R. Bulsara, S. B. Lowen, C. D. Rees, Cooperative behavior in the periodically modulated Wiener process: noise-induced complexity in a model neuron. Phys. Rev. E 49,4989-5000, 1994. 
[2] A. R. Bulsara, T. C. Elston, C. R. Doering, S. B. Lowen, K. Lindenberg, Cooperative behavior in periodically driven noisy integrate-fire models of neuronal dynamics. Phys. Rev. E 53, 3958-3969, 1996.

[3] S. E. Fienberg, Stochastic models for single neuron firing trains: a survey. Biometrics 30, 399-427, 1974.

[4] G. L Gerstein, B. B. Mandelbrot, Random walk models for the spike activity of a single neuron. Biophys. $J$. 4,41-68, 1964.

[5] H. C. Tuckwell, Stochactic Process in the Neurosciences, Society for industrial and Applied Mathematics, Philadelphia, PA, 1989.

[6] S. Kammer, A general first-passage-time model for multivariate credit spreads and a note on barrier option pricing ,Ph.D Thesis, Justus-Liebig-Universit at Giessen, 8-20, 2007.

[7] H. Li, M. Shaked, On the first passage times for Markov processes with monotone convex transition kernels , Stochastic Processes and their Applications, 58(2): 205-206,1995.

[8] M. Mandel, Estimating disease progression using panel data, Biostatistics, 11(2): 1-13,2010.

[9] A. Ridder, Asymptotic optimality of the cross-entropy method for Markov chain problems, Procedia Computer Science, 1(1): 1565-1572,2010.

[10] J.P. Tian, D. Kannan, Lumpability and Commutativity of Markov Processes, Stochastic Analysis and Applications; 24(3):685-702, 2006.

[11] F.S.Hillier, G.J. Lieberman, Introduction to operations research, Seventh Edition, Mcgraw-Hill, 2001

[12] E. Çınlar, Introduction to Stochastic Processes, Englewood Cliffs, New Jersey, 106-277, 1997.

[13] D. Gupta, Fundamentals of Probability: A first course ,Springer Texts in Statistics, 671-672, 2010.

[14] L. Zhang, Lumpability Approximation Methods for Markov Models, Ph.D. Thesis, Purdue University, 6-16, 27-39, 2006.

[15] J.G. Kemeny, J.L. Snell, Finite Markov Chains, Springer, Berlin, 124-132, 1976.

[16] A. Alzaatreh, C.Lee, F. Famoye, On the discrete analogues of continuous distributions, Statistical Methodology, 9: 589-603, 2012. 